\title{
Recurrence Relations for Moments of Upper Record Values from Two Parameters Rayleigh Distribution
}

Submitted by

\author{
Mohamed Salah Ahmed Hassanein \\ Demonstrator at Department of Statistics and Insurance \\ Faculty of Commerce - Zagazig University
}




\section{Introduction}

Rayleigh distribution is a special case of the two parameter Weibull distribution with the shape parameter. The Rayleigh distribution has a wide range of applications including life testing experiments and clinical studies. This distribution can be considered one of the famous distribution that are useful in several areas of statistics including life testing and reliability which age with time as its failure rate is a linear function of time. John William was a British physicist who discovered the element argon, which earned him the Nobel Prize for Physics. He also discovered 'Rayleigh scattering' and predicted the existence of the surface waves now known as Rayleigh waves. This statistical model was first introduced by Rayleigh (1880). Siddiqui (1962) discussed the origin and properties of the Rayleigh distribution. The origin and other aspects of this distribution can be found in Siddiqui (1962). Polovko (1968) noted its importance in electro vacuum devices. Dyer and Whisenand (1973) demonstrated the importance of this distribution in communication engineering. Cliff and Ord (1975) showed that Rayleigh distribution arises as the distribution of the distance between an individual . Bhattacharya and Tyagi (1990) mentioned that in some clinical studies dealing with cancer patients, the survival pattern follows Rayleigh distribution . Many researchers have studied the Rayleigh distribution, its characterizations and inferences based on it because of its wide applicability in biological sciences, economics, electronics, engineering, fisheries, geography, modeling and analysis of life time data, oceanography, pharmacology, physics, reliability, wireless communication system. For detailed treatment on Rayleigh distribution, the interested readers are referred to Johnson et al. (1994) and references therein. 
Record values and the associated statistics are of interest and important in many real life applications, such as: weather, educations, industry, economic, and sports data. The statistical study of record values started with Chandler (1952) and spread in different directions. Record values are defined as a model for successive extremes. Chandler (1952) introduced the study of record values and documented many of basic properties of record values. Feller (1966) gave some examples of record values with respect to gambling problems. He presented some examples of record values in gambling problems and studied problems of characterizations based on record values and order statistics. Resnick (1987) discussed the asymptotic theory of records and followed in seventies by Nagaraja (1989) and Ahsanullah (1979). Properties of record values have been extensively studied in the literature Ahsanullah (1988), Balakrishnan (1989) and Nagaraja (1978). Balakrishnan and Ahsanullah (1994) established some recurrence relations for the single and double moments of lower record values from Gumble distribution. Nagaraja (1978) provides the basic results on the topic of moments. Moments of record values are of great interest in the general theory of linear estimation and prediction in location and scale families such as discussed by Ashanullah (1988), Nagaraja (1988) . Pawlas , P and Szynal ,D.(1997) obtain analogous results of k- record statistics. Probability distributions based on the moments of record values by Balakrishnan et al (1994) .Balakrishnan and Ahsanullah (1994) establish some recurrence relations for single and product moments of record values from Gumbel and Generalized pareto distribution, respectively.

Recurrence relations and identities are often helpful to determine moments of order statistics and record values. Thus in the literature some relations for single and product moments of record values from exponential, 
Weibull, Pareto, generalized pareto, uniform and other distributions have been discussed by Arnold ( 1989 ) and Balakrishnan (1989). Ashanullah (1995) discussed this topics applied on power function distribution that he used recurrence relations method to compute all the single and product moments under record valued. Kamps (1994) discussed recurrence relations of this type and a characteristics results are given for a class of probability distributions. Ashanullah (1993) discussed this topics applied on Generalized Pareto distribution that he used recurrence relations method to compute all the single and product moments under record valued. Balakrishnan (1993) discussed this topics applied on exponential distribution using recurrence relation method to compute all the single and product moments under upper record values . Ashanullah, Balakrishnan (1993) discussed this topics applied on Generalized Extreme Value then they used recurrence relation method to compute all the single and product moments under upper record values. Balakrishnan (1994) has derived some recurrence relations by the single and product moments of order statistics from the right truncated exponential distribution. Balakrishnan and Ahsanullah (1994) established some recurrence relations of single and double moments of record values from the Lomax distribution. Balakrishnan and Ahsanullah (1995) established some recurrence relations of single and double moments of upper record values from exponential distribution. Pawlas and Szynal (2000) derived recurrence relations satisfied by single and double moments of the kth upper record values from the Pareto, generalized Pareto and Burr distributions. .Pawlas and Szynal (2001) derived recurrence relations for single and double moments of GOSs under the concept from Pareto, generalized Pareto and Burr distributions. 
The two parameters Rayleigh distribution is a continuous probability distribution that plays an important in real life applications

Probability density function of Rayleigh distribution with two parameters is given by

$f(x \backslash a, b)=2 a(x-b) \exp \left(-a(x-b)^{2}\right) b<x<\infty, a>0, b>0$

where (a) is the scale parameter, (b) is location parameter and cumulative distribution is given by

$$
F(x)=1-\exp \left(-a(x-b)^{2}\right)
$$

In which case, we have $\frac{f(x)}{2 a(x-b)}=1-F(x)$

Let $x_{1}, x_{2}, x_{3}, \ldots \ldots, x_{n}$ be sequence of independent and identically distributed random variables with cumulative distribution function and probability density function .set $x_{n}=\max \left(x_{(1)}<x_{(2)}<x_{(3)}<\ldots . .<x_{(n)}\right)$.we say $x_{n}$ is an upper record value of this sequence if $x_{i}>x_{i-1}, n>1, i>1$ and $x_{1}$ is an lower record value

Probability density function of upper record values is given by

$f_{n}(x)=\frac{1}{\Gamma(n)}\{R(x)\}^{n-1} f(x),, b<x<\omega$

where $R(x)=-\operatorname{In}(1-F(x)$

\section{See (Ahsanullah 1993 )}

where $\mathrm{x}$ has Rayleigh distribution with two parameters, Probability density 
function of upper record values from Rayleigh distribution with two parameters is given by :

$$
\begin{aligned}
& f_{n}(x)=\frac{1}{\Gamma(n)}\left\{-\operatorname{In}\left(\exp \left(-a(x-b)^{2}\right)\right\}^{n-1} 2 a(x-b) \exp \left(-a(x-b)^{2}\right)\right. \\
& =\frac{2 a(x-b)}{\Gamma(n)}\{a\}^{n-1}\{(x-b)\}^{2 n-2} \exp \left(-a(x-b)^{2}\right)
\end{aligned}
$$

This function can be rewritten as the following :

$$
f_{n}(x)=\frac{2 a^{n}(x-b)^{2 n-1}}{\Gamma(n)} \exp \left(-a(x-b)^{2}\right)
$$

Putting $b=0$, One can find that

$f_{n}(x)=\frac{2 a^{n}(x)^{2 n-1}}{\Gamma(n)} \exp \left(-a(x)^{2}\right)$

\section{Single Moments of Upper Record Values}

The single moments can be computed of upper record values from Rayleigh with two parameters, Some single moments of upper record values can be derived as follows

$$
E\left(x_{u(n)}^{r}\right)=\int_{b}^{\infty} x^{r} f_{n}(x) d x=\frac{2(a)^{n}}{\Gamma(n)} \int_{b}^{\infty} x^{r}(x-b)^{2 n-1} \exp \left(-a(x-b)^{2}\right) d x
$$

Substituting

$$
\begin{aligned}
& a(x-b)^{2}=z \quad \text { Then } \quad d x=\frac{d z}{2 \sqrt{a} \sqrt{z}} \\
& E\left(x_{u(n)}^{r}\right)=\frac{1}{\Gamma(n)} \int_{0}^{\infty} \sum_{i=0}^{r}\left(i^{r}\right)\left(\frac{\sqrt{z}}{\sqrt{a}}\right)^{i}(b)^{r-i}(z)^{n-1} \exp (-z) d z \text { for } \mathrm{r}=1,2,3,4, \ldots \ldots
\end{aligned}
$$

The first four moments can be written as :

The first zero moment 
$E\left(x_{u(n)}\right)=\left[b+\frac{\phi(n)}{\sqrt{a}}\right]$ Where $\phi(n)=\frac{\Gamma\left(n+\frac{1}{2}\right)}{\Gamma(n)}$

The second zero moment

$$
E\left(x_{u(n)}^{2}\right)=\left[\frac{a b^{2}+2 \sqrt{a} b \varphi(n)+n}{a}\right]
$$

The third zero moment

$$
E\left(x_{u(n)}^{3}\right)=\left[\frac{b^{3} a^{\frac{3}{2}}+\left(n+\frac{1}{2}\right) \varphi(n)+3 a^{\frac{1}{2}} b n+3 a b^{2} \varphi(n)}{a^{\frac{3}{2}}}\right]
$$

The fourth zero moment

$$
E\left(x_{u(n)}^{4}\right)=\left(\frac{n(n+1)+4 \sqrt{a}(b)\left(n+\frac{1}{2}\right) \varphi(n)+6 a n(b)^{2}+4 a^{\frac{3}{2}}(b)^{3} \varphi(n)+a^{2}(b)^{4}}{a^{2}}\right)
$$

Using the previous equations, the four central moments can be calculated as the following The second central moment can be computed using the following relation

$$
\mu_{2}=\left[\frac{n-(\phi(n))^{2}}{a}\right]
$$

Since $\mu_{2}$ must be positive then $(\phi(n))^{2}<n$, one can be notice that $\mu_{2}$ is dependent on (a) and independent on (b).

Using the following relation, the third central moment can be calculated as the following 


$$
\mu_{3}=\left[\frac{\varphi(n)\left[\left[\frac{1}{2}-2 n\right]+2(\varphi(n))^{2}\right]}{a^{\frac{3}{2}}}\right]
$$

Using the following relation, the four central moments can be calculated as the following:

$$
\mu_{4}=\frac{n(n+1)-3(\varphi(n))^{4}+(\varphi(n))^{2}\left(3 a b^{2}+2 n-2\right)}{a^{2}}
$$

Measure of Skewness and Kurtosis

The measure of Skewness and Kurtosis can be written as : $\beta_{1}=\frac{\mu_{3}}{\sqrt{\mu_{2}{ }^{3}}}$

Previously, The researcher showed that $\phi(n)<\sqrt{n}$

Since the numerator of coefficient of skewness is $2(\phi(n))^{2}-\left(2 n-\frac{1}{2}\right)>0$

This means that the distribution is Negative .

Since the coefficient of kurtosis is given by

$$
\beta_{2}=\frac{n(n+1)-3(\phi(n))^{4}+(\phi(n))^{2}\left\{3 a b^{2}+2 n-2\right\}}{\left(n-(\phi(n))^{2}\right)^{2}}
$$

Since

$$
n(n+1)-3(\phi(n))^{4}+(\phi(n))^{2}\left\{3 a b^{2}+2 n-2\right\}>3\left(n-(\phi(n))^{2}\right)^{2}
$$

This means that the distribution is peaked .

\section{Product Moments of Upper Record Values}

The product moments of upper record values from Rayleigh with one and two parameters based on two upper record values are considered .

Let $x_{u(1)}, x_{u(2)}, x_{u(3)}, \ldots . . x_{u(n)}$ and $x_{u(1)}, x_{u(2)}, x_{u(3)}, \ldots \ldots \ldots, x_{u(m)}$ be the first $\mathrm{n}$ and $\mathrm{m}$ 
upper record values where $\mathrm{m}<\mathrm{n}$.

The joint distribution between two upper record values is given by $f_{m, n}(x, y)=f\left(x_{u(m)}, x_{u(n)}\right)=\frac{1}{\Gamma(m) \Gamma(n-m)}\left[-\operatorname{In}(1-F(x)]^{m-1} \frac{f(x)}{1-F(x)}\left[-\operatorname{In}\left(1-F(y)+\operatorname{In}(1-F(x)]^{n-m-1} f(y)\right.\right.\right.$

\section{See (Ahsanullah 1993 )}

Using Rayleigh distribution with two parameters, the previous equation can be rewritten as the following

$$
\begin{aligned}
& f_{m, n}(x, y)=c\left[a(x-b)^{2}\right]^{m-1} 2 a(x-b)\left[a(y-b)^{2}-a(x-b)^{2}\right]^{n-m-1} 2 a(y-b) \exp \left(-a(y-b)^{2}\right) \\
& \qquad c=\frac{1}{\Gamma(m) \Gamma(n-m)} \\
& \text { where }
\end{aligned}
$$

$$
f_{m, n}(x, y)=4(a)^{n} c(y-b)(x-b)^{2 m-1}\left[(y-b)^{2}-(x-b)^{2}\right]^{n-m-1} \exp \left(-a(y-b)^{2}\right)
$$

The product moments of two upper record values is given by

$$
\begin{aligned}
& E\left(x^{r}, y^{s}\right)=c \int_{-\infty}^{\infty} \int_{-\infty}^{\infty} x^{r} y^{s}\left[-\operatorname{In}(1-F(x)]^{m-1} \frac{f(x)}{1-F(x)}\left[-\operatorname{In}\left(1-F(y)+\operatorname{In}(1-F(x)]^{n-m-1} f(y) d x d y\right.\right.\right. \\
& =c \int_{-\infty}^{\infty} y^{s} \boldsymbol{I}(y) f(y) d y
\end{aligned}
$$

where

$$
I(y)=\int_{y}^{\infty} x^{r}\left[-\operatorname{In}(1-F(x)]^{m-1} \frac{f(x)}{1-F(x)}\left[-\operatorname{In}\left(1-F(y)+\operatorname{In}(1-F(x)]^{n-m-1} d x\right.\right.\right.
$$

\section{See (Ahsanullah 1995 )}

The product moments can be computed as follows :

$$
\mu_{m, n}^{(r, s)}=E\left(x_{u(m)}^{r}, x_{u(n)}^{s}\right)=c \int_{-\infty}^{\infty} y^{s} I(y) f(y) d y
$$


Using the substitution $a(x-b)^{2}=z$ and binomial theory, this equation can be rewritten as the following:

$$
I(y)=2(a)^{n-1} \int_{0}^{\infty}\left(\frac{\sqrt{z}}{\sqrt{a}}+b\right)^{r}\left(\frac{\sqrt{z}}{\sqrt{a}}\right)^{2 m}\left[(y-b)^{2}-\left(\frac{\sqrt{z}}{\sqrt{a}}\right)^{2}\right]^{n-m-1} \frac{d z}{2 \sqrt{a} \sqrt{z}}
$$

Then

$=\frac{1}{\sqrt{a}} \int_{0}^{\infty}\left(\frac{\sqrt{z}}{\sqrt{a}}+b\right)^{r} z^{m-\frac{1}{2}}\left[a(y-b)^{2}-z\right]^{n-m-1} d z$

Then

$$
I(y)=\frac{1}{\sqrt{a}} \int_{0}^{\infty} z^{m-\frac{1}{2}} \sum_{i=0}^{r}\left(i^{r}\right)\left(\frac{\sqrt{z}}{\sqrt{a}}\right)^{i}(b)^{r-i} \sum_{j=0}^{n-m-1}\left(j^{n-m-1}\right)(-1)^{j}(y-b)^{2 j}(a)^{j}(z)^{n-m-1-j} d z
$$

Using the substitution $a(y-b)^{2}=L$ and binomial theory

The four product moments can be computed as the following

$$
E\left(x^{r}, y^{s}\right)=a^{\frac{5}{2}} c \int_{b}^{\infty}\left(\frac{\sqrt{L}}{\sqrt{a}}+b\right)^{s}\left[\int_{0}^{\infty} z^{m-\frac{1}{2}} \sum_{i=0}^{r}\left(i^{r}\right)\left(\frac{\sqrt{z}}{\sqrt{a}}\right)^{i}(b)^{r-i} \sum_{j=0}^{n-m-1}\left(j^{n-m-1}\right)(-1)^{j}(L)^{j}(z)^{n-m-1-j} d z\right] \exp (-L) d L
$$

then

$$
E\left(x^{r}, y^{s}\right)=a^{\frac{5}{2}} c \int_{0}^{\infty} \sum_{k=0}^{s}\left(k^{s}\right)\left(\frac{\sqrt{L}}{\sqrt{a}}\right)^{k}(b)^{s-k} \exp (-L) d L\left[\int_{0}^{\infty} z^{m-\frac{1}{2}} \sum_{i=0}^{r}\left(i^{r}\right)\left(\frac{\sqrt{z}}{\sqrt{a}}\right)^{i}(b)^{r-i} \sum_{j=0}^{n-m-1}\left(j^{n-n-1}\right)(-1)^{j}(L)^{j}(z)^{n-m-1-j} d z\right]
$$

\section{Recurrence Relations for Single Moments}

The recurrence relations method can be used in simple way to compute all the simple moments of all record values. Once again using integration by parts, some simple recurrence relations for single moments of upper record values can be derived as the following :

Some simple recurrence relations satisfied by single moments of upper record 
values are given as the following $E\left(x_{u(n)}^{r}\right)=\frac{1}{\Gamma(n)} \int_{-\infty}^{\infty} x^{r}\{R(x)\}^{n-1} f(x) d x$

the first moment upon integration by parts can be computed as the following $=\frac{1}{\Gamma(n)}\left[\frac{1}{(r+1)} \int_{-\infty}^{\infty}\{-\operatorname{In}(1-F(x))\}^{n-1} f(x) x^{r+1} d x-\frac{(n-1)}{(r+1)} \int_{-\infty}^{\infty}\{-\operatorname{In}(1-F(x))\}^{n-2} x^{r+1} \frac{-f(x)}{-(1-F(x))} f(x) d x\right]$ where $\frac{f(x)}{1-F(x)}=2 a(x-b)$

$$
E\left(x_{u(n)}^{r+1}\right)=(r+1) E\left(x_{u(n)}^{r}\right)+2 a E\left(x_{u(n-1)}^{r+2}\right)-2 a b E\left(x_{u(n-1)}^{r+1}\right)
$$

Then by repeated applying the recurrence relation, The second moment from the first moment also upon integration by parts can be computed as the following

$$
\begin{aligned}
& E\left(x_{u(n)}^{r+1}\right)=\frac{1}{\Gamma(n)} \int_{-\infty}^{\infty} x^{r+1}[-\operatorname{In}(1-F(x))]^{n-1} f(x) d x \\
& =\frac{1}{\Gamma(n)}\left[\frac{1}{(r+2)} \int_{-\infty}^{\infty}\{-\operatorname{In}(1-F(x))\}^{n-1} f(x) x^{r+2} d x-\frac{(n-1)}{(r+2)} \int_{-\infty}^{\infty}\{-\operatorname{In}(1-F(x))\}^{n-2} x^{r+2} \frac{-f(x)}{-(1-F(x))} f(x) d x\right] \\
& E\left(x_{u(n)}^{r+2}\right)=(r+2) E\left(x_{u(n)}^{r+1}\right)+2 a E\left(x_{u(n-1)}^{r+3}\right)-2 a b E\left(x_{u(n-1)}^{r+2}\right)
\end{aligned}
$$

The third moment form the second moment upon integration by parts can be computed as the following

$$
\begin{aligned}
& E\left(x_{u(n)}^{r+2}\right)=\frac{1}{\Gamma(n)} \int_{-\infty}^{\infty} x^{r+2}[-\operatorname{In}(1-F(x))]^{n-1} f(x) d x \\
& =\frac{1}{\Gamma(n)}\left[\frac{1}{(r+3)} \int_{-\infty}^{\infty} x^{r+3}[-\operatorname{In}(1-F(x))]^{n-1} f(x) d x-\frac{(n-1)}{(r+3)} \int_{-\infty}^{\infty}[-\operatorname{In}(1-F(x))]^{n-2} \frac{-f(x)}{-(1-F(x))} x^{r+3} f(x) d x\right]
\end{aligned}
$$




$$
E\left(x_{u(n)}^{r+3}\right)=(r+3) E\left(x_{u(n)}^{r+2}\right)+2 a E\left(x_{u(n-1)}^{r+4}\right)-2 a b E\left(x_{u(n-1)}^{r+3}\right)
$$

the four moments from th third moment upon integration by parts can be found as the following:

$$
\begin{aligned}
& E\left(x_{u(n)}^{r+3}\right)=\frac{1}{\Gamma(n)} \int_{-\infty}^{\infty} x^{r+3}[-\operatorname{In}(1-F(x))]^{n-1} f(x) d x \\
& =\frac{1}{\Gamma(n)}\left[\frac{1}{(r+4)} \int_{-\infty}^{\infty} x^{r+4}[-\operatorname{In}(1-F(x))]^{n-1} f(x) d x-\frac{(n-1)}{(r+4)} \int_{-\infty}^{\infty}[-\operatorname{In}(1-F(x))]^{n-2} \frac{-f(x)}{-(1-F(x))} x^{r+4} f(x) d x\right] \\
& E\left(x_{u(n)}^{r+4}\right)=(r+4) E\left(x_{u(n)}^{r+3}\right)+2 a E\left(x_{u(n-1)}^{r+5}\right)-2 a b E\left(x_{u(n-1)}^{r+4}\right)
\end{aligned}
$$

\section{Recurrence Relations for Product Moments}

In the following, the researcher used an alternative method for calculating product moment called as the recurrence relation under Rayleigh distribution with two parameters

Since the general case for the product moments is given by

$$
E\left(x^{r}, y^{s}\right)=c_{m, n-m} \int_{-\infty}^{\infty} x^{r}\left[-\operatorname{In}(1-F(x)]^{m-1} \frac{f(x)}{1-F(x)} d x \int_{-\infty}^{\infty} y^{s}\left[-\operatorname{In}\left(1-F(y)+\operatorname{In}(1-F(x)]^{n-m-1} f(y) d y\right.\right.\right.
$$

Using integration by parts, the $(\mathrm{r}+1)$ product moment can be computed as the following:

$$
\begin{aligned}
& E\left(x^{r}, y^{s}\right)=c_{m, n-m}\left(\int _ { - \infty } ^ { \infty } x ^ { r + 1 } \frac { 1 } { ( r + 1 ) } \left[-\ln (1-F(x)]^{m-1} \frac{f(x)}{1-F(x)} d x-\frac{(m-1)}{(r+1)} \int_{-\infty}^{\infty}\left[-\ln (1-F(x)]^{m-2} \frac{f(x) f(x)}{(1-F(x))(1-F(x))} x^{r+1} d x\right)\right.\right. \\
& \left(\int _ { - \infty } ^ { \infty } y ^ { s + 1 } \frac { 1 } { ( s + 1 ) } \left[-\ln \left(1-F(y)+\ln (1-F(x)]^{n-m-1} f(y) d y-\frac{(n-m-1)}{(s+1)} \int_{-\infty}^{\infty}\left[-\ln \left(1-F(y)+\ln (1-F(x)]^{n-m-2} \frac{f(y) f(x)}{(1-F(y))-(1-F(x))} y^{s+1} d y\right)\right.\right.\right.\right. \\
& \text { Where, } \\
& c_{m-k, n-m-k}=\frac{1}{\Gamma(m-k) \Gamma(n-m-k)}, \text { for k=0,1,2,.... }
\end{aligned}
$$


Using Rayleigh distribution with two parameters, the general product moments can be rewritten as the following :

$$
\begin{aligned}
& E\left(x^{r} y^{s}\right)=\frac{1}{(r+1)(s+1)} E\left(x_{u(m)}^{r+1} y_{u(n-m)}^{s+1}\right)-\frac{(n-m-1)}{(r+1)(s+1)} E\left(x_{u(m)}^{r+1} y_{u(n-m)}^{s+1}\right) \\
& -\frac{2 a(m-1)}{(r+1)(s+1)} E\left(x_{u(m-1)}^{r+2} y_{u(n-m)}^{s+1}\right)-\frac{2 a b^{2}(m-1)}{(r+1)(s+1)} E\left(x_{u(m-1)}^{r} y_{u(n-m)}^{s+1}\right) \\
& +\frac{4 a b(m-1)}{(r+1)(s+1)} E\left(x_{u(m-1)}^{r+1} y_{u(n-m)}^{s+1}\right)+\frac{2 a(m-1)(n-m-1)}{(r+1)(s+1)} E\left(x_{u(m-1)}^{r+2} y_{u(n-m-1)}^{s+1}\right) \\
& +\frac{2 a b^{2}(m-1)(n-m-1)}{(r+1)(s+1)} E\left(x_{u(m-1)}^{r} y_{u(n-m-1)}^{s+1}\right)-\frac{4 a b(m-1)(n-m-1)}{(r+1)(s+1)} E\left(x_{u(m-1)}^{r+1} y_{u(n-m-1)}^{s+1}\right)
\end{aligned}
$$

In case of $\mathrm{n}=\mathrm{m}+1, \mathrm{th}(\mathrm{r}+1)$ product moment can be rewritten as the following

$$
\left.E\left(x_{u(m)}^{r+1}\right)_{u(n-m)}^{s+1}\right)=L_{1}\left[E\left(x_{u(m-1)}^{r+2} y_{u(n-m)}^{s+1}\right)+b^{2} E\left(x_{u(m-1)}^{r} y_{u(n-m)}^{s+1}\right)-2 b E\left(x_{u(m-1)}^{r+1} y_{u(n-m)}^{s+1}\right)\right]
$$

Where

$$
L_{1}=\frac{1}{3}(r+1)(s+1) E\left(x^{r} y^{s}\right)+2 a(m-1)
$$

From equation above, the $(\mathrm{r}+2)$ product moments from th $(\mathrm{r}+1)$ product moment using integration by parts can be computed as the following:

$$
E\left(x^{r+1}, y^{s+1}\right)=c_{m, n-m} \int_{-\infty}^{\infty} x^{r+1}\left[-\operatorname{In}(1-F(x)]^{m-1} \frac{f(x)}{1-F(x)} d x \int_{-\infty}^{\infty} y^{s+1}\left[-\operatorname{In}\left(1-F(y)+\operatorname{In}(1-F(x)]^{n-m-1} f(y) d y\right.\right.\right.
$$

this equation can be rewritten as the following :

$$
\begin{aligned}
& E\left(x^{r+1}, y^{s+1}\right)=c_{m, n-m}\left(\int _ { - \infty } ^ { \infty } x ^ { r + 2 } \frac { 1 } { ( r + 2 ) } \left[-\ln (1-F(x)]^{m-1} \frac{f(x)}{1-F(x)} d x-\frac{(m-1)}{(r+2)} \int_{-\infty}^{\infty}\left[-\ln (1-F(x)]^{m-2} \frac{f(x) f(x)}{(1-F(x))(1-F(x))} x^{r+2} d x\right)\right.\right. \\
& \left(\int _ { - \infty } ^ { \infty } y ^ { s + 2 } \frac { 1 } { ( s + 2 ) } \left[-\ln \left(1-F(y)+\ln (1-F(x)]^{n-m-1} f(y) d y-\frac{(n-m-1)}{(s+2)} \int_{-\infty}^{\infty}\left[-\ln \left(1-F(y)+\ln (1-F(x)]^{n-m-2} \frac{f(y) f(x)}{(1-F(y))-(1-F(x))} y^{s+2} d y\right)\right.\right.\right.\right.
\end{aligned}
$$


Using Rayleigh distribution with two parameters, the general product moment can be rewritten as the following:

$$
\begin{aligned}
& E\left(x^{r+1} y^{s+1}\right)=\frac{1}{(r+2)(s+2)} E\left(x_{u(m)}^{r+2} y_{u(n-m)}^{s+2}\right)-\frac{(n-m-1)}{(r+2)(s+2)} E\left(x_{u(m)}^{r+2} y_{u(n-m)}^{s+2}\right) \\
& -\frac{2 a(m-1)}{(r+2)(s+2)} E\left(x_{u(m-1)}^{r+3} y_{u(n-m)}^{s+2}\right)-\frac{2 a b^{2}(m-1)}{(r+2)(s+2)} E\left(x_{u(m-1)}^{r+1} y_{u(n-m)}^{s+2}\right) \\
& +\frac{4 a b(m-1)}{(r+2)(s+2)} E\left(x_{u(m-1)}^{r+2} y_{u(n-m)}^{s+2}\right)+\frac{2 a(m-1)(n-m-1)}{(r+2)(s+2)} E\left(x_{u(m-1)}^{r+3} y_{u(n-m-1)}^{s+2}\right) \\
& +\frac{2 a b^{2}(m-1)(n-m-1)}{(r+2)(s+2)} E\left(x_{u(m-1)}^{r+1} y_{u(n-m-1)}^{s+2}\right)-\frac{4 a b(m-1)(n-m-1)}{(r+2)(s+2)} E\left(x_{u(m-1)}^{r+2} y_{u(n-m-1)}^{s+2}\right)
\end{aligned}
$$

Then, th( $\mathrm{r}+2)$ product moment can be rewritten as the following :

$$
\left.E\left(x_{u(m)}^{r+2} y_{u(n-m)}^{s+2}\right)=L_{2}\left[E\left(x_{u(m-1)}^{r+3} y_{u(n-m)}^{s+2}\right)+b^{2} E\left(x_{u(m-1)}^{r+1}\right)_{u(n-m)}^{s+2}\right)-2 b E\left(x_{u(m-1)}^{r+2} y_{u(n-m)}^{s+2}\right)\right]
$$

Where

$$
L_{2}=\frac{1}{3}(r+2)(s+2) E\left(x^{r+1} y^{s+1}\right)+2 a(m-1)
$$

from the previous equation, th $(\mathrm{r}+3)$ product moment can be computed as the following:

$$
E\left(x^{r+2}, y^{s+2}\right)=c_{m, n-m} \int_{-\infty}^{\infty} x^{r+2}\left[-\operatorname{In}(1-F(x)]^{m-1} \frac{f(x)}{1-F(x)} d x \int_{-\infty}^{\infty} y^{s+2}\left[-\operatorname{In}\left(1-F(y)+\operatorname{In}(1-F(x)]^{n-m-1} f(y) d y\right.\right.\right.
$$

then

$$
\begin{aligned}
& E\left(x^{r+2}, y^{s+2}\right)=c_{m, n-m}\left(\int _ { - \infty } ^ { \infty } x ^ { r + 3 } \frac { 1 } { ( r + 3 ) } \left[-\ln (1-F(x)]^{m-1} \frac{f(x)}{1-F(x)} d x-\frac{(m-1)}{(r+3)} \int_{-\infty}^{\infty}\left[-\ln (1-F(x)]^{m-2} \frac{f(x) f(x)}{(1-F(x))(1-F(x))} x^{r+3} d x\right)\right.\right. \\
& \left(\int _ { - \infty } ^ { \infty } y ^ { s + 3 } \frac { 1 } { ( s + 3 ) } \left[-\ln \left(1-F(y)+\ln (1-F(x)]^{n-m-1} f(y) d y-\frac{(n-m-1)}{(s+3)} \int_{-\infty}^{\infty}\left[-\ln \left(1-F(y)+\operatorname{In}(1-F(x)]^{n-m-2} \frac{f(y) f(x)}{(1-F(y))-(1-F(x))} y^{s+3} d y\right)\right.\right.\right.\right.
\end{aligned}
$$

Using Rayleigh distribution with two parameters, this equation can be rewritten 
as the following:

$$
\begin{aligned}
& E\left(x^{r+2} y^{s+2}\right)=\frac{1}{(r+3)(s+3)} E\left(x_{u(m)}^{r+3} y_{u(n-m)}^{s+3}\right)-\frac{(n-m-1)}{(r+3)(s+3)} E\left(x_{u(m)}^{r+3} y_{u(n-m)}^{s+3}\right) \\
& -\frac{2 a(m-1)}{(r+3)(s+3)} E\left(x_{u(m-1)}^{r+4} y_{u(n-m)}^{s+3}\right)-\frac{2 a b^{2}(m-1)}{(r+3)(s+3)} E\left(x_{u(m-1)}^{r+2} y_{u(n-m)}^{s+3}\right) \\
& +\frac{4 a b(m-1)}{(r+3)(s+3)} E\left(x_{u(m-1)}^{r+3} y_{u(n-m)}^{s+3}\right)+\frac{2 a(m-1)(n-m-1)}{(r+3)(s+3)} E\left(x_{u(m-1)}^{r+4} y_{u(n-m-1)}^{s+3}\right) \\
& +\frac{2 a b^{2}(m-1)(n-m-1)}{(r+3)(s+3)} E\left(x_{u(m-1)}^{r+2} y_{u(n-m-1)}^{s+3}\right)-\frac{4 a b(m-1)(n-m-1)}{(r+3)(s+3)} E\left(x_{u(m-1)}^{r+3} y_{u(n-m-1)}^{s+3}\right)
\end{aligned}
$$

th $(\mathrm{r}+3)$ product moment can be constracted as the following :

$$
\left.\left.\left.\left.E\left(x_{u(m)}^{r+3}\right)_{u(n-m)}^{s+3}\right)=L_{3}\left[E\left(x_{u(m-1)}^{r+4}\right)_{u(n-m)}^{s+3}\right)+b^{2} E\left(x_{u(m-1)}^{r+2}\right)_{u(n-m)}^{s+3}\right)-2 b E\left(x_{u(m-1)}^{r+3}\right)_{u(n-m)}^{s+3}\right)\right]
$$

Where

$$
L_{3}=\frac{1}{3}(r+3)(s+3) E\left(x^{r+2} y^{s+2}\right)+2 a(m-1)
$$

also th $(\mathrm{r}+4)$ product moment can be constracted as the following:

$$
E\left(x^{r+3}, y^{s+3}\right)=c_{m, n-m} \int_{-\infty}^{\infty} x^{r+3}\left[-\operatorname{In}(1-F(x)]^{m-1} \frac{f(x)}{1-F(x)} d x \int_{-\infty}^{\infty} y^{s+3}\left[-\operatorname{In}\left(1-F(y)+\operatorname{In}(1-F(x)]^{n-m-1} f(y) d y\right.\right.\right.
$$

Then

$$
\begin{aligned}
& E\left(x^{r+3}, y^{s+3}\right)=c_{m, n-m}\left(\int _ { - \infty } ^ { \infty } x ^ { r + 4 } \frac { 1 } { ( r + 4 ) } \left[-\ln (1-F(x)]^{m-1} \frac{f(x)}{1-F(x)} d x-\frac{(m-1)}{(r+4)} \int_{-\infty}^{\infty}\left[-\ln (1-F(x)]^{m-2} \frac{f(x) f(x)}{(1-F(x))(1-F(x))} x^{r+4} d x\right)\right.\right. \\
& \left(\int _ { - \infty } ^ { \infty } y ^ { s + 4 } \frac { 1 } { ( s + 4 ) } \left[-\ln \left(1-F(y)+\ln (1-F(x)]^{n-m-1} f(y) d y-\frac{(n-m-1)}{(s+4)} \int_{-\infty}^{\infty}\left[-\ln \left(1-F(y)+\ln (1-F(x)]^{n-m-2} \frac{f(y) f(x)}{(1-F(y))-(1-F(x))} y^{s+4} d y\right)\right.\right.\right.\right.
\end{aligned}
$$

Using Rayleigh distribution with two parameters, this equation can be rewritten as the following 


$$
\begin{aligned}
& E\left(x^{r+3} y^{s+3}\right)=\frac{1}{(r+4)(s+4)} E\left(x_{u(m)}^{r+4} y_{u(n-m)}^{s+4}\right)-\frac{(n-m-1)}{(r+4)(s+4)} E\left(x_{u(m)}^{r+4} y_{u(n-m)}^{s+4}\right) \\
& -\frac{2 a(m-1)}{(r+4)(s+4)} E\left(x_{u(m-1)}^{r+5} y_{u(n-m)}^{s+4}\right)-\frac{2 a b^{2}(m-1)}{(r+4)(s+4)} E\left(x_{u(m-1)}^{r+3} y_{u(n-m)}^{s+4}\right) \\
& +\frac{4 a b(m-1)}{(r+4)(s+4)} E\left(x_{u(m-1)}^{r+4} y_{u(n-m)}^{s+4}\right)+\frac{2 a(m-1)(n-m-1)}{(r+4)(s+4)} E\left(x_{u(m-1)}^{r+5} y_{u(n-m-1)}^{s+4}\right) \\
& +\frac{2 a b^{2}(m-1)(n-m-1)}{(r+4)(s+4)} E\left(x_{u(m-1)}^{r+3} y_{u(n-m-1)}^{s+4}\right)-\frac{4 a b(m-1)(n-m-1)}{(r+4)(s+4)} E\left(x_{u(m-1)}^{r+4} y_{u(n-m-1)}^{s+4}\right)
\end{aligned}
$$

Finally, th $(\mathrm{r}+4)$ product moment between two upper record values can be rewritten as the following

$$
\left.\left.\left.E\left(x_{u(m)}^{r+4} y_{u(n-m)}^{s+4}\right)=L_{4}\left[E\left(x_{u(m-1)}^{r+5}\right)_{u(n-m)}^{s+4}\right)-(n-m-1) E\left(x_{u(m-1)}^{r+5}\right)_{u(n-m-1)}^{s+4}\right)+b^{2} E\left(x_{u(m-1)}^{r+3}\right)_{u(n-m)}^{s+4}\right)\right]
$$

Where

$$
L_{4}=\frac{1}{3}(r+4)(s+4) E\left(x^{r+3} y^{s+3}\right)+2 a(m-1)
$$

\section{Conclusions}

In this paper the following points are discussed

1- Single and product moments of upper record values from Rayleigh distribution with two parameters using recurrence relations method.

2- The recurrence relations for single and product moments from two parameters Rayleigh distribution

3- The coefficient of skewness and Kurtosis . 


\section{Reference}

1- Ahsanullah, M. (1988). Introduction to Record Values. Ginn Press، Needham Heights, Massachusetts Vol (2)

2- Ahsanullah ,M and Balakrishnan ,N (1994). Recurrence Relations for Single and Product Moments of Record Values from Generalized Pareto Distribution .Communication Statistics, Theory Mathematics, Vol 23(10). 3- Balakrishnan and Ahsanullah (1993).Recurrence relations for moments of record values from generalized extreme value distribution, Communications in Statistics - Theory and Methods, Vol (22).

4- Balakrishnan ,N and Chan,P.S (1993). Record Values from Rayleigh and Weibull distribution and associated inference, National institute of standards and Technology,Journal of Research ,Special Publications Vol (66) .

5- Balakrishnan ,N-Chan ,P.S and Ahsanullah ,M (1993) .Recurrence relations for Moments of Record Values from Generalized Extreme Value Distribution .Communication Statistics Vol 22(5), Rider University, USA and McMaster University , Canada.

6- Balakrishnan and Ahsanullah (1994). Recurrence relations for single and product moments of record values from generalized Pareto distribution. Communications in Statistics - Theory and Methods,Vol( 23). 7- Balakrishnan, $N$ and Ahsanullah ,M(1995). Relations for Single and Product Moments of Record Values from Exponential Distribtuion.Journal of Applied Statistical Science, Vol (3), Rider University , USA.

8- Balakrishnan ,N , Ahsanullah, $M$ and Chan,P.S (1995). Relations for single and produnct moments of record values from Gumbel distribution , Statist . Probab.Lett, Vol (15). 
9- Balakrishnan ,N and Sandhu,R.A (1995) . Recurrence Relations for Single and Product Moments of Order Statistics from a generalized Half Logistic Distribution With Application to Inference . J.Statistics.Comput .Simul , Vol(52), Canada.

10- Chandler ,K.N.(1952) .The distribution and frequency of record values ,J.Roy.Statist.Soc,Ser .Vol (15).

11- David , H.A (1981) .Order Statistics .Second Edition .John Wiley and Sons ,Inc Vol (2), New York, USA.

12- Hohsin ,M(2009). Recurrence Relations for Single and Product Moments of Record Values from Erlang -Truncated Exponentail Distribution World Applied Sciences Journal Vol 6(2), Communication Statistics ,Institute of Information Technology ,Lahor Pakistan

13- Khan , R.U and Benazir, Z (2009) .Recurrence Relations for single and product moments of record values from Gompertz distribution and characterization .World Applied Sciences Journal7,Vol(10),India

14- Nain, $K$ (2010 ): Recurrence Relations for Single and Product Moments of kth Record Values from Generalized Weibull distribution and a Characterization, International Mathematical Forum, Vol (5).

15- Pawlas , P and Szynal ,D.(1997) .Recurrence Relations of K-The Record Values from Pareto, Generalized Pareto and Burr Distribtuions‘Maria Curie -Sktodowska University ,Pl.M.Curie Sktodowska, Vol 26(2).

16- Singh ,S.K and Saran ,J (2008) .Recurrence Relations for Single and Product Moments of K-Th Record Values from Linear Exponential Distribution and Characterization .Asian Journal of Mathematics of Statistics,Vol (3) University of Delhi ,India

17- Saran, J. and Kumari ,T (2013). Recurrence relations for marginal and joint moment generating functions of upper k-record values from Gompertz distribution, ProbStat Forum is an e-journal, Volume (6). 
